\title{
Integer solutions of a sequence of decomposable form inequalities
}

\author{
by
}

K. GYÖRY (Debrecen) and Min Ru (Houston, TX)

1. Introduction. Let $F(\mathbf{X})=F\left(X_{0}, \ldots, X_{m}\right) \in \mathbb{Z}[\mathbf{X}]$ be a decomposable form, i.e. a homogeneous polynomial which factorizes into linear forms over $\overline{\mathbb{Q}}$. Assume that $q=\operatorname{deg} F>2 m$, and consider the decomposable form inequality

$$
0<|F(\mathbf{x})|<|\mathbf{x}|^{\nu} \quad \text { in } \mathbf{x}=\left(x_{0}, \ldots, x_{m}\right) \in \mathbb{Z}^{m+1},
$$

where $|\mathbf{x}|=\max _{0 \leq i \leq m}\left|x_{i}\right|$ and $\nu<q-2 m$. For $m=1$, it follows from Roth's approximation theorem (cf. [Sch2], p. 120) that if the linear factors of $F$ are pairwise nonproportional, then (1.1) has only finitely many solutions. Using his subspace theorem, W. M. Schmidt ([Sch1], [Sch2]) generalized this to arbitrary $m$, under the assumptions that (i) any $m+1$ of the linear factors of $F$ are linearly independent over $\overline{\mathbb{Q}}$, and that (ii) $F$ is not divisible by a form with rational coefficients of degree less than $m+1$. Later H. P. Schlickewei [Schl] extended this theorem to the case when the ground ring is an arbitrary finitely generated subring of $\mathbb{Q}$. These results have obvious applications to decomposable form equations of the form

$$
F(\mathbf{x})=G(\mathbf{x}) \quad \text { in } \mathbf{x} \in \mathbb{Z}^{m+1},
$$

where $G \in \mathbb{Z}[\mathbf{X}]$ is a nonzero polynomial of degree $<q-2 m$ (cf. [Sch2]). In the important special case when $G$ is a constant, the first author [Gy1] proved the finiteness of the number of solutions of (1.2) under the assumption (i) only, which is necessary in general. For the case when $G$ is a constant, there are also more general finiteness results: see [Sch2], [EGy2], [EGy4] and the references given there. The above mentioned results have further appli-

1991 Mathematics Subject Classification: Primary 11J68; Secondary 11J25.

The first author was supported in part by the Hungarian Academy of Sciences and by Grants 16975 and 25157 from the Hungarian National Foundation for Scientific Research. The second author was supported in part by NSF grant DMS-9800361. 
cations to resultant inequalities and resultant equations (cf. [Sch1], [Sch2], [Schl], [Gy1], [Gy3]).

The purpose of this paper is to improve and generalize the above results of [Sch1], [Sch2], [Schl] to a sequence of decomposable form inequalities over number fields (cf. Theorem 2). For a single decomposable form inequality, Theorem 2 implies the finiteness of the number of solutions of (1.1) without assuming (ii) (cf. Theorem 1). Our Theorems 1 and 2 do not remain valid in general for $\nu=q-2 m$. As a consequence of Theorem 2, finiteness results are established for decomposable form equations of the form (1.2) over number fields (cf. Theorems 3,4). Some applications are also given to resultant inequalities (Theorems 5,6). Finally, we generalize our results to the case where $q>2 m-l+1$ with $1 \leq l \leq m+1$, by showing that in this situation the set of solutions is contained in a finite union of subspaces of dimension at most $l$ (cf. Theorems 7-9).

2. Notation and statement of the main results. Let $\mathbb{K}$ be an algebraic number field. Denote by $\mathbb{M}(\mathbb{K})$ the set of places of $\mathbb{K}$ and write $\mathbb{M}_{\infty}(\mathbb{K})$ for the set of archimedean places of $\mathbb{K}$. For $v \in \mathbb{M}(\mathbb{K})$ denote by ||$_{v}$ the associated absolute value, normalized so that ||$_{v}=||$ (standard absolute value) on $\mathbb{Q}$ if $v$ is archimedean, whereas for $v$ nonarchimedean $|p|_{v}=p^{-1}$ if $v$ lies above the rational prime $p$. Denote by $\mathbb{K}_{v}$ the completion of $\mathbb{K}$ with respect to $v$ and by $d_{v}=\left[\mathbb{K}_{v}: \mathbb{Q}_{v}\right]$ the local degree. We put \|\|$_{v}=||_{v}^{d_{v}}$.

For $\mathbf{x}=\left(x_{0}, \ldots, x_{m}\right) \in \mathbb{K}^{m+1}$, we put $\|\mathbf{x}\|_{v}=\max _{0 \leq i \leq m}\left\|x_{i}\right\|_{v}$ and we denote by

$$
h(\mathbf{x})=\frac{1}{[\mathbb{K}: \mathbb{Q}]} \sum_{v \in \mathbb{M}(\mathbb{K})} \log \|\mathbf{x}\|_{v}
$$

the absolute logarithmic height of $\mathbf{x}$. Given a polynomial $P$ with coefficient in $\mathbb{K}$, we define $\|P\|_{v}$ and $h(P)$ as the \|\|$_{v}$-value and absolute logarithmic height, respectively, of the point whose coordinates are the coefficients of $P$. As is known, $h(\mathbf{x})$ and $h(P)$ are independent of the choice of the field $\mathbb{K}$. Further, $h(\lambda \mathbf{x})=h(\mathbf{x})$ and $h(\lambda P)=h(P)$ for all $\lambda \in \overline{\mathbb{Q}}^{*}$.

Let $S$ be a finite subset of $\mathbb{M}(\mathbb{K})$ containing $\mathbb{M}_{\infty}(\mathbb{K})$. An element $x \in \mathbb{K}$ is said to be $S$-integer if $\|x\|_{v} \leq 1$ for each $v \in \mathbb{M}(\mathbb{K})-S$. Denote by $O_{S}$ the set of $S$-integers. The units of $O_{S}$ are called $S$-units. They form a multiplicative group which is denoted by $O_{S}^{*}$. For $\alpha \in \mathbb{K}-\{0\}$, let $N_{S}(\alpha)=\prod_{v \in S}\|\alpha\|_{v}$ denote the $S$-norm of $\alpha$. If $\alpha \in O_{S}-\{0\}$ then $N_{S}(\alpha)$ is a positive integer and $N_{S}(\alpha)=1$ for $\alpha \in O_{S}^{*}$. For $\mathbf{x}=\left(x_{0}, \ldots, x_{m}\right) \in \mathbb{K}^{m+1}$, define the $S$-height as $H_{S}(\mathbf{x})=\prod_{v \in S}\|\mathbf{x}\|_{v}$. If $\mathbf{x} \in O_{S}^{m+1}-\{0\}$, then $H_{S}(\mathbf{x}) \geq 1$ and $H_{S}(\alpha \mathbf{x})=H_{S}(\mathbf{x})$ for $\alpha \in O_{S}^{*}$. For a polynomial $P$ with coefficients in $\mathbb{K}$, let $H_{S}(P)$ denote the $S$-height of that point whose coordinates are the coefficients of $P$. 
Linear forms $L_{1}, \ldots, L_{q} \in \overline{\mathbb{Q}}\left[x_{0}, \ldots, x_{m}\right]$ are said to be in general position if any $m+1$ of them are linearly independent over $\overline{\mathbb{Q}}$.

Let $q$ and $m$ denote positive integers such that $q>2 m$, and let $F(\mathbf{X})=$ $F\left(X_{0}, \ldots, X_{m}\right) \in O_{S}[\mathbf{X}]$ be a decomposable form of degree $q$. For given real numbers $c$ and $\nu$ with $c>0$, consider the solutions of the inequality

$$
0<N_{S}(F(\mathbf{x})) \leq c H_{S}(\mathbf{x})^{\nu} \quad \text { in } \mathbf{x} \in O_{S}^{m+1} .
$$

If $\mathbf{x}$ is a solution of $(2.1)$, then so is $\mathbf{x}^{\prime}=\eta \mathbf{x}$ for every $\eta \in O_{S}^{*}$. Such solutions $\mathbf{x}, \mathbf{x}^{\prime}$ are called $O_{S^{-}}^{*}$ proportional.

THEOREM 1. Suppose that $\nu<q-2 m$ and that the linear factors of $F$ over $\overline{\mathbb{Q}}$ are in general position. Then (2.1) has only finitely many $O_{S}^{*}$-nonproportional solutions.

REMARK 1. Theorem 1 does not remain valid in general for $\nu=q-$ $2 m$ (cf. Schmidt [Sch1]). In the important case when $\mathbb{K}=\mathbb{Q}$, Theorem 1 provides an improvement of the previous results of Schmidt [Sch1, Sch2] and Schlickewei $\left[\right.$ Schl]. Further, for $\mathbb{K}=\mathbb{Q}, S=\mathbb{M}_{\infty}(\mathbb{Q})$ and $m=1$, Theorem 1 gives Theorem 3B in Chapter V of Schmidt [Sch2].

Remark 2. For $\nu=0$, Theorem 1 was proved in a quantitative form in Györy [Gy3]. For a generalization considered over an arbitrary finitely generated ground ring over $\mathbb{Z}$, see Győry [Gy1].

The main result of this paper is Theorem 2. Theorem 1 is an immediate consequence.

TheOREM 2. Let $q, m$ be positive integers with $q>2 m$. Let $c, \nu$ be real numbers with $c>0, \nu<q-2 m$ and $\mathbb{G}$ a finite extension of $\mathbb{K}$. For $n=1,2, \ldots$, let $F_{n}(\mathbf{X})=F_{n}\left(X_{0}, \ldots, X_{m}\right) \in O_{S}[\mathbf{X}]$ denote a decomposable form of degree $q$ which factorizes into linear factors over $\mathbb{G}$, and suppose that these factors are in general position for each $n$. Then there does not exist an infinite sequence of $O_{S}^{*}$-nonproportional $\mathbf{x}_{n} \in O_{S}^{m+1}, n=1,2, \ldots$, for which

$$
0<N_{S}\left(F_{n}\left(\mathbf{x}_{n}\right)\right) \leq c H_{S}\left(\mathbf{x}_{n}\right)^{\nu} \quad \text { for } n=1,2, \ldots,
$$

and

$$
h\left(F_{n}\right)=o\left(h\left(\mathbf{x}_{n}\right)\right) \quad \text { if } h\left(\mathbf{x}_{n}\right) \rightarrow \infty \text { as } n \rightarrow \infty .
$$

3. Proof of Theorem 2. We keep the notation of Section 2 and recall Schmidt's subspace theorem with moving targets proved by Min $\mathrm{Ru}$ and P. Vojta (see $[\mathrm{RV}]$ ). The fixed target case, i.e. the case when $L_{j, n}=L_{j}$ for each $j$ and $n$, is due to Min Ru and P. M. Wong (see Theorem 4.1 in [RW]).

Theorem A [RV]. Given linear forms $L_{1, n}, \ldots, L_{q, n} \in \mathbb{K}\left[X_{0}, \ldots, X_{m}\right]$, $n=1,2, \ldots$, in general position for each $n$ and a sequence $\mathbf{x}_{n} \in \mathbb{K}^{m+1}$ such 
that for $j=1, \ldots, q, L_{j, n}\left(\mathbf{x}_{n}\right) \neq 0$ and $h\left(L_{j, n}\right)=o\left(h\left(\mathbf{x}_{n}\right)\right)$ as $n \rightarrow \infty$. Then, for any $\varepsilon>0$, there exists an infinite subsequence $\left\{n_{k}\right\} \subseteq \mathbb{N}$ such that

$$
\frac{1}{[\mathbb{K}: \mathbb{Q}]} \sum_{v \in S} \sum_{j=1}^{q} \log \frac{\left\|\mathbf{x}_{n_{k}}\right\|_{v} \cdot\left\|L_{j, n_{k}}\right\|_{v}}{\left\|L_{j, n_{k}}\left(\mathbf{x}_{n_{k}}\right)\right\|_{v}} \leq(2 m+\varepsilon) h\left(\mathbf{x}_{n_{k}}\right) \quad \text { for all } k .
$$

The above statement is contained in the second part of Theorem 3.1 in [RV]. Note that, due to a printing mistake, the term $(2 m+\varepsilon) h\left(\mathbf{x}_{n_{k}}\right)$ on the right-hand side of the above inequality was incorrectly stated as $(2 m+1+\varepsilon) h\left(\mathbf{x}_{n_{k}}\right)$ in $[\mathrm{RV}]$.

Proof of Theorem 2. We shall prove Theorem 2 by using the above theorem of [RV]. Assume that there is an infinite sequence $\mathbf{x}_{n}=\left(x_{0, n}, \ldots, x_{m, n}\right)$ $\in O_{S}^{m+1}$ which satisfies (2.2). First consider the case when the values $h\left(\mathbf{x}_{n}\right)$ are bounded. We may assume without loss of generality that $x_{0, n} \neq 0$ for each $n$. Then the $h\left(\mathbf{x}_{n} / x_{0, n}\right)$ are bounded and this implies that $\mathbf{x}_{n} / x_{0, n}$ may assume only finitely many values in $\mathbb{K}^{m+1}$. Hence there are infinitely many $n$ such that $\mathbf{x}_{n}=x_{0, n} \mathbf{x}_{0}$ for some $\mathbf{x}_{0} \in \mathbb{K}^{m+1}$. For these $n$ we deduce from (2.2) that

$$
0<N_{S}\left(x_{0, n}\right)^{q} N_{S}\left(F_{n}\left(\mathbf{x}_{0}\right)\right) \leq c N_{S}\left(x_{0, n}\right)^{\nu} H_{S}\left(\mathbf{x}_{0}\right)^{\nu}
$$

and hence $N_{S}\left(x_{0, n}\right)$ are bounded. Since $x_{0, n} \in O_{S}$, it follows (see e.g. [EGY3]) that there are infinitely many $n$ for which $x_{0, n}=\eta_{n} x_{0}^{\prime}$ with some $\eta_{n} \in O_{S}^{*}$ and fixed $x_{0}^{\prime} \in O_{S}$. This implies that for these $n$ the $\mathbf{x}_{n}$ considered above are $O_{S}^{*}$-proportional, which is a contradiction.

Next consider the case when $h\left(\mathbf{x}_{n}\right)$ are not bounded. We may assume that $h\left(\mathbf{x}_{n}\right) \rightarrow \infty$ as $n \rightarrow \infty$. Then, by assumption, (2.3) also holds. Further, it follows that $H_{S}\left(\mathbf{x}_{n}\right) \rightarrow \infty$ as $n \rightarrow \infty$. For $n=1,2, \ldots$, let $F_{n}=L_{1, n} \ldots L_{q, n}$ be a factorization of $F_{n}$ over $\mathbb{G}$ into linear factors. Then by Proposition 2.4 of Chapter 3 in $[\mathrm{L}]$ it follows that

$$
\max _{j} h\left(L_{j, n}\right) \leq h\left(F_{n}\right)+c_{1}
$$

where $c_{1}$ is a positive constant which depends only on $q, m$ and $\mathbb{G}$. Together with (2.3) this gives

$$
\max _{j} h\left(L_{j, n}\right)=o\left(h\left(\mathbf{x}_{n}\right)\right) \quad \text { as } n \rightarrow \infty .
$$

Let $\mathbb{M}(\mathbb{G})$ denote the set of places of $\mathbb{G}$. For $v \in \mathbb{M}(\mathbb{G})$, define and normalize \|\|$_{v}$ as over $\mathbb{K}$ above. Further, let $T$ denote the set of extensions to $\mathbb{G}$ of the places in $S$. Then we deduce from (2.2) that

(3.2) $0<N_{T}\left(F_{n}\left(\mathbf{x}_{n}\right)\right)=N_{S}\left(F_{n}\left(\mathbf{x}_{n}\right)\right)^{[\mathbb{G}: \mathbb{K}]} \leq\left(c H_{S}\left(\mathbf{x}_{n}\right)^{\nu}\right)^{[\mathbb{G}: \mathbb{K}]}=c_{2} H_{T}\left(\mathbf{x}_{n}\right)^{\nu}$, where $c_{2}=c^{[\mathbb{G}: \mathbb{K}]}$. Here $N_{T}(), H_{T}()$ are defined over $\mathbb{G}$ in the same way as $N_{S}(), H_{S}()$ over $\mathbb{K}$. 
Let $\varepsilon>0$ with $0<\varepsilon<q-2 m-\nu$. Then by the above Theorem A of $[\mathrm{RV}]$, there is an infinite subsequence $\mathbf{x}_{n_{k}} \in O_{S}^{m+1}, k=1,2, \ldots$, of $\left\{\mathbf{x}_{n}\right\}$, which, without loss of generality, we assume to be $\left\{\mathbf{x}_{n}\right\}$ itself, such that

$$
\frac{1}{[\mathbb{G}: \mathbb{Q}]} \sum_{v \in T} \sum_{j=1}^{q} \log \frac{\left\|\mathbf{x}_{n}\right\|_{v} \cdot\left\|L_{j, n}\right\|_{v}}{\left\|L_{j, n}\left(\mathbf{x}_{n}\right)\right\|_{v}} \leq(2 m+\varepsilon) h\left(\mathbf{x}_{n}\right) .
$$

On the other hand, $F_{n}\left(\mathbf{x}_{n}\right)=\prod_{j=1}^{q} L_{j, n}\left(\mathbf{x}_{n}\right)$. Furthermore, in view of $\mathbf{x}_{n} \in O_{S}^{m+1}$, we have

$$
h\left(\mathbf{x}_{n}\right) \leq \frac{1}{[\mathbb{G}: \mathbb{Q}]} \log H_{T}\left(\mathbf{x}_{n}\right)
$$

Hence

$$
\prod_{v \in T} \frac{\left\|\mathbf{x}_{n}\right\|_{v}^{q} \cdot \prod_{j=1}^{q}\left\|L_{j, n}\right\|_{v}}{\left\|F_{n}\left(\mathbf{x}_{n}\right)\right\|_{v}} \leq H_{T}\left(\mathbf{x}_{n}\right)^{2 m+\varepsilon}
$$

whence

$$
\frac{H_{T}^{q}\left(\mathbf{x}_{n}\right) \cdot \prod_{v \in T} \prod_{j=1}^{q}\left\|L_{j, n}\right\|_{v}}{N_{T}\left(F_{n}\left(\mathbf{x}_{n}\right)\right)} \leq H_{T}\left(\mathbf{x}_{n}\right)^{2 m+\varepsilon} .
$$

Since the coefficients of $L_{j, n}$ are $T$-integers,

$$
\prod_{v \in T} \prod_{j=1}^{q}\left\|L_{j, n}\right\|_{v} \geq 1 \quad \text { for } n=1,2, \ldots
$$

Furthermore, it follows from (3.2) that

$$
N_{T}\left(F_{n}\left(\mathbf{x}_{n}\right)\right) \leq c_{2} H_{T}\left(\mathbf{x}_{n}\right)^{\nu} \quad \text { for } n=1,2, \ldots
$$

Combining (3.4)-(3.6) gives

$$
H_{T}\left(\mathbf{x}_{n}\right)^{q} \leq c_{2} H_{T}\left(\mathbf{x}_{n}\right)^{\nu+2 m+\varepsilon} .
$$

Since $H_{T}\left(\mathbf{x}_{n}\right) \rightarrow \infty$ as $n \rightarrow \infty$, and $q>\nu+2 m+\varepsilon$, this is a contradiction.

4. Consequences of Theorem 2. In this section, we give four further consequences of our Theorem 2 .

First, it is easy to see that Theorem 2 implies the following result concerning the $S$-integer solutions of a sequence of decomposable form equations of the form (1.2).

TheOREM 3. Given positive integers $q, m$ with $q>2 m$, a finite extension $\mathbb{G}$ of $\mathbb{K}$, and a sequence of polynomials $G_{n}(\mathbf{X}) \in O_{S}[\mathbf{X}]$ in $\mathbf{X}=$ $\left(X_{0}, \ldots, X_{m}\right)$ such that $\operatorname{deg}\left(G_{n}(\mathbf{X})\right)<q-2 m$ for $n=1,2, \ldots$ Let $F_{n}(\mathbf{X})=$ $F_{n}\left(X_{0}, \ldots, X_{m}\right) \in O_{S}[\mathbf{X}]$ be a sequence of decomposable forms of degree $q$ 
such that $F_{n}$ factorizes into linear forms over $\mathbb{G}$ which are in general position for each $n$. Then there does not exist an infinite sequence of $O_{S}^{*}$-nonproportional $\mathbf{x}_{n} \in O_{S}^{m+1}$ for which

$$
\begin{gathered}
F_{n}\left(\mathbf{x}_{n}\right)=G_{n}\left(\mathbf{x}_{n}\right) \neq 0, \quad n=1,2, \ldots, \\
\log H_{S}\left(G_{n}\right)=o\left(\log H_{S}\left(\mathbf{x}_{n}\right)\right) \quad \text { if } H_{S}\left(\mathbf{x}_{n}\right) \rightarrow \infty \text { as } n \rightarrow \infty
\end{gathered}
$$

and

$$
h\left(F_{n}\right)=o\left(h\left(\mathbf{x}_{n}\right)\right) \quad \text { if } h\left(\mathbf{x}_{n}\right) \rightarrow \infty \text { as } n \rightarrow \infty .
$$

REMARK 3. We note that under the assumptions (4.2) and (4.3), equations (4.1) may have infinitely many $O_{S}^{*}$-proportional solutions $\mathbf{x}_{n}$. Indeed, this is the case if $F_{n}$ is the same for each $n, \eta_{n}$ is an infinite sequence of $S$-units, $\mathbf{x}_{0} \in O_{S}^{m+1}$ with $F_{n}\left(\mathbf{x}_{0}\right)=1, G_{n}=\eta_{n}^{q}$ and $\mathbf{x}_{n}=\eta_{n} \cdot \mathbf{x}_{0}$ for $n=1,2, \ldots$

Proof (of Theorem 3). Suppose that there is an infinite sequence of $O_{S}^{*}$-nonproportional $\mathbf{x}_{n} \in O_{S}^{m+1}$ satisfying the conditions of Theorem 3 . It is easy to see that the $H_{S}\left(\mathbf{x}_{n}\right)$ are not bounded. We may assume that $H_{S}\left(\mathbf{x}_{n}\right) \rightarrow \infty$ as $n \rightarrow \infty$. It follows that

$$
\begin{aligned}
0 & <N_{S}\left(F_{n}\left(\mathbf{x}_{n}\right)\right)=N_{S}\left(G_{n}\left(\mathbf{x}_{n}\right)\right)=\prod_{v \in S}\left\|G_{n}\left(\mathbf{x}_{n}\right)\right\|_{v} \\
& \leq c_{3} \prod_{v \in S}\left(\left\|G_{n}\right\|_{v} \cdot\left\|\mathbf{x}_{n}\right\|_{v}^{\operatorname{deg} G_{n}}\right)=c_{3} H_{S}\left(G_{n}\right) H_{S}\left(\mathbf{x}_{n}\right)^{\operatorname{deg} G_{n}}
\end{aligned}
$$

where $c_{3}$ is a positive constant which depends only on $q$ and $\mathbb{G}$. We choose $\nu$ such that $q-2 m-1<\nu<q-2 m$. Then, by (4.2), we deduce that

$$
H_{S}\left(G_{n}\right) \leq H_{S}\left(\mathbf{x}_{n}\right)^{\nu-\operatorname{deg} G_{n}} \quad \text { as } n \rightarrow \infty .
$$

Hence (4.4) implies that

$$
0<N_{S}\left(F_{n}\left(\mathbf{x}_{n}\right)\right) \leq c_{3} H_{S}\left(\mathbf{x}_{n}\right)^{\nu} \quad \text { as } n \rightarrow \infty,
$$

which contradicts Theorem 2 .

We deduce from Theorem 3 the following.

TheOREM 4. Given positive integers $q, m$ with $q>2 m$, and a polynomial $G(\mathbf{X}) \in O_{S}[\mathbf{X}]$ in $\mathbf{X}=\left(X_{0}, \ldots, X_{m}\right)$ with total degree less than $q-2 m$. Let $F(\mathbf{X}) \in O_{S}[\mathbf{X}]$ be a decomposable form whose linear factors are in general position. Then the equation

$$
F(\mathbf{X})=G(\mathbf{X})
$$

has only finitely many solutions $\mathbf{x}=\left(x_{0}, \ldots, x_{m}\right) \in O_{S}^{m+1}$ with $G(\mathbf{x}) \neq 0$.

Remark 4. For $\mathbb{K}=\mathbb{Q}, S=\mathbb{M}_{\infty}(\mathbb{Q})$ and $m=1$, our Theorem 4 gives the second assertion of Theorem 3B of [Sch2, Ch. V]. When $G$ is constant, Theorem 4 was proved over more general ground rings in K. Györy [Gy1]. 
Pr o of (of Theorem 4). Suppose that (4.5) has infinitely many solutions $\mathbf{x}$ with $G(\mathbf{x}) \neq 0$. Then, by Theorem 3 , there are also infinitely many solutions $\mathbf{x}$ such that $\mathbf{x}=\eta \mathbf{x}_{0}$ with $\eta \in O_{S}^{*}$ and with some fixed $\mathbf{x}_{0} \in O_{S}^{m+1}$. Then it follows from (4.5) that

$$
\eta^{q} F\left(\mathbf{x}_{0}\right)=G\left(\eta \mathbf{x}_{0}\right) .
$$

This can be regarded as an equation of degree $q$ in $\eta$ with leading coefficient $F\left(\mathbf{x}_{0}\right) \neq 0$. However, this equation has at most $q$ solutions in $\eta$, which proves the assertion.

Let $q, m$ be positive integers with $q>2 m$, and let $P \in O_{S}[X]$ be a polynomial of degree $q$ without multiple zeros. For given $c>0$ and $\nu$, consider the solutions of the resultant inequality

$$
0<N_{S}(\operatorname{Res}(P, Q)) \leq c H_{S}(Q)^{\nu} \quad \text { in } Q \in O_{S}[X] \text { of degree } m .
$$

If $Q$ is a solution then so is $\eta Q$ for each $\eta \in O_{S}^{*}$. Such solutions $Q, \eta Q$ are called $O_{S}^{*}$-proportional.

TheOREM 5. If $\nu<q-2 m$, then (4.6) has only finitely many $O_{S}^{*}$-nonproportional solutions.

Remark 5 . For $\mathbb{K}=\mathbb{Q}$, Theorem 5 is an improvement of previous results of E. Wirsing [W], W. M. Schmidt [Sch1] and H. P. Schlickewei [Schl]. Theorem 5 does not remain valid when $\nu=q-2 m$ (cf. [Sch1]). For $\nu=0$, Theorem 5 was proved in a quantitative form in K. Győry [Gy2]. For a generalization to polynomials over more general ground rings, see K. Győry [Gy1].

Theorem 5 is an immediate consequence of the next theorem which will be deduced from Theorem 2 .

THEOREM 6. Let $q, m$ be positive integers with $q>2 m, c, \nu$ real numbers with $c>0, \nu<q-2 m$, and $\mathbb{G}$ a finite extension of $\mathbb{K}$. For every integer $n \geq 1$, let $P_{n} \in O_{S}[X]$ denote a polynomial of degree $q$ with distinct zeros in $\mathbb{G}$. Then there does not exist a sequence of $O_{S}^{*}$-nonproportional $Q_{n} \in$ $O_{S}[X]$ with $\operatorname{deg} Q_{n}=m$ for which

$$
0<N_{S}\left(\operatorname{Res}\left(P_{n}, Q_{n}\right)\right) \leq c H_{S}\left(Q_{n}\right)^{\nu}, \quad n=1,2, \ldots,
$$

and

$$
h\left(P_{n}\right)=o\left(h\left(Q_{n}\right)\right) \quad \text { if } h\left(Q_{n}\right) \rightarrow \infty \text { as } n \rightarrow \infty .
$$

This should be compared with Corollary 4 of K. Győry [Gy2] where $\nu=0$, but $O_{S}$ is replaced by a more general ground ring.

Proof (of Theorem 6). Put

$$
P_{n}(X)=a_{0, n}\left(X-\alpha_{1, n}\right) \ldots\left(X-\alpha_{q, n}\right) \quad \text { for } n=1,2, \ldots
$$


Assume that there is an infinite sequence of

$$
Q_{n}(X)=x_{0, n} X^{m}+x_{1, n} X^{m-1}+\ldots+x_{m, n},
$$

satisfying (4.7) and (4.8). For $n \geq 1$ set

$$
F_{n}(\mathbf{X})=F_{n}\left(X_{0}, \ldots, X_{m}\right)=a_{0, n}^{m} \prod_{i=1}^{q}\left(X_{0} \alpha_{i, n}^{m}+X_{1} \alpha_{i, n}^{m-1}+\ldots+X_{m}\right) .
$$

Then $F_{n}$ has its coefficients in $O_{S}$. Further, it factorizes into linear factors over $\mathbb{G}$, and these are in general position for every $n$. For $\mathbf{x}_{n}=\left(x_{0, n}, \ldots, x_{m, n}\right)$ we have

$$
\operatorname{Res}\left(P_{n}, Q_{n}\right)=F_{n}\left(\mathbf{x}_{n}\right)
$$

Hence (4.7) implies that

$$
0<N_{S}\left(F_{n}\left(\mathbf{x}_{n}\right)\right) \leq c H_{S}\left(Q_{n}\right)^{\nu}=c H_{S}\left(\mathbf{x}_{n}\right)^{\nu} .
$$

Further, by using Proposition 2.4 of Chapter 3 in [L] it is easy to see that

$$
h\left(F_{n}\right) \leq q h\left(P_{n}\right)+c_{4},
$$

where $c_{4}$ is a constant which depends only on $q, m$ and $\mathbb{G}$. Hence if $h\left(Q_{n}\right)$ $\rightarrow \infty$ as $n \rightarrow \infty$, it follows from (4.10) and (4.8) that

$$
h\left(F_{n}\right)=o\left(h\left(\mathbf{x}_{n}\right)\right) \quad \text { if } h\left(\mathbf{x}_{n}\right) \rightarrow \infty \text { as } n \rightarrow \infty .
$$

Together with (4.9) this contradicts our Theorem 2.

5. Some generalizations. In the previous sections, we assume that $q>2 m$ where $q$ is the degree of a decomposable form $F$, and $m+1$ is the number of variables in $F$. In this section, we consider the case $q>2 m-l+1$, where $l$ is an integer with $1 \leq l \leq m+1$. As we indicated earlier, for $l>1$, no finiteness result is expected. Rather, we show that the set of solutions is then contained in a finite union of proper subspaces.

First of all, Theorem 1 can be generalized as follows.

TheOREM 7. Let $q, m, l$ denote positive integers with $q>2 m-l+1,1 \leq$ $l \leq m+1$, and $\mathbb{G}$ a finite extension of $\mathbb{K}$. Let $F(\mathbf{X})=F\left(X_{0}, \ldots, X_{m}\right) \in$ $O_{S}[\mathbf{X}]$ be a decomposable form of degree $q$ which factorizes into linear factors over $\mathbb{G}$. For given real numbers $c, \nu$ with $c>0$, consider the solutions of the inequality

$$
0<N_{S}(F(\mathbf{x})) \leq c H_{S}(\mathbf{x})^{\nu} \quad \text { in } \mathbf{x} \in O_{S}^{m+1} .
$$

Suppose that $\nu<q-2 m+l-1$ and that the linear factors of $F$ over $\overline{\mathbb{Q}}$ are in general position. Then the set of solutions of (5.1) is contained in a finite union of linear subspaces of $\mathbb{K}^{m+1}$ of dimension at most $l$.

It is easy to see that for $l=1$, Theorem 7 gives Theorem 1 . For $\nu=0$, compare Theorem 3 of [EGy1]. 
The proof of Theorem 7 is similar to the proof of Theorem 2. First we recall Theorem 4.1 of $\mathrm{Ru}-$ Wong [RW] (see also [RV], Theorem 3.1).

TheOREM B (Theorem 4.1 of [RW]). Given linear forms $L_{1}, \ldots, L_{q} \in$ $\mathbb{K}\left[X_{0}, \ldots, X_{m}\right]$ in general position. Then for any $\varepsilon>0$, the set of points $\mathbf{x} \in \mathbb{K}^{m+1}$ such that $L_{j}(\mathbf{x}) \neq 0$ for $j=1, \ldots, q$ and

$$
\frac{1}{[\mathbb{K}: \mathbb{Q}]} \sum_{v \in S} \sum_{j=1}^{q} \log \frac{\|\mathbf{x}\|_{v} \cdot\left\|L_{j}\right\|_{v}}{\left\|L_{j}(\mathbf{x})\right\|_{v}} \geq(2 m-l+1+\varepsilon) h(\mathbf{x})
$$

is contained in a finite union of linear subspaces of $\mathbb{K}^{m+1}$ of dimension at most l.

Proof (of Theorem 7). Let $\mathbf{x} \neq \mathbf{0}$ be a solution of (5.1). Let $F=$ $L_{1} \ldots L_{q}$ be a factorization of $F$ over $\mathbb{G}$ into linear factors. Let $\mathbb{M}(\mathbb{G})$ denote the set of places of $\mathbb{G}$. For $v \in \mathbb{M}(\mathbb{G})$, define and normalize \|\|$_{v}$ as over $\mathbb{K}$ above. Further, let $T$ denote the set of extensions to $\mathbb{G}$ of the places in $S$. Then we deduce from (5.1) that

$$
0<N_{T}(F(\mathbf{x}))=N_{S}(F(\mathbf{x}))^{[\mathbb{G}: \mathbb{K}]} \leq\left(c H_{S}(\mathbf{x})^{\nu}\right)^{[\mathbb{G}: \mathbb{K}]}=c_{2} H_{T}(\mathbf{x})^{\nu},
$$

where $c_{2}=c^{[\mathbb{G}: \mathbb{K}]}$. Here $N_{T}(), H_{T}()$ are defined over $\mathbb{G}$ in the same way as $N_{S}(), H_{S}()$ over $\mathbb{K}$. We have $F(\mathbf{x})=\prod_{j=1}^{q} L_{j}(\mathbf{x})$. It follows that

$$
\begin{aligned}
\prod_{v \in T} \frac{\|\mathbf{x}\|_{v}^{q} \cdot \prod_{j=1}^{q}\left\|L_{j}\right\|_{v}}{\prod_{j=1}^{q}\left\|L_{j}(\mathbf{x})\right\|_{v}} & =\frac{H_{T}(\mathbf{x})^{q} \cdot\left(\prod_{v \in T} \prod_{j=1}^{q}\left\|L_{j}\right\|_{v}\right)}{N_{T}(F(\mathbf{x}))} \\
& \geq \frac{H_{T}(\mathbf{x})^{q} \cdot\left(\prod_{v \in T} \prod_{j=1}^{q}\left\|L_{j}\right\|_{v}\right)}{c_{2} H_{T}(\mathbf{x})^{\nu}} \\
& =c_{5} H_{T}(\mathbf{x})^{q-\nu},
\end{aligned}
$$

where $c_{5}=\prod_{v \in T} \prod_{j=1}^{q}\left\|L_{j}\right\|_{v} / c_{2}$. In view of $\mathbf{x} \in O_{T}^{m}$, we have

$$
h(\mathbf{x}) \leq \frac{1}{[\mathbb{G}: \mathbb{Q}]} \log H_{T}(\mathbf{x}) .
$$

Thus, by taking logarithms on both sides of (5.2),

$$
\frac{1}{[\mathbb{G}: \mathbb{Q}]} \sum_{v \in T} \sum_{j=1}^{q} \log \frac{\|\mathbf{x}\|_{v} \cdot\left\|L_{j}\right\|_{v}}{\left\|L_{j}(\mathbf{x})\right\|_{v}} \geq(q-\nu) h(\mathbf{x})+c_{6},
$$

where $c_{6}=\log c_{5} /[\mathbb{G}: \mathbb{Q}]$. Let $\varepsilon>0$ with $q-\nu \geq 2 m+l-1>2 \varepsilon$. Then

$$
\frac{1}{[\mathbb{G}: \mathbb{Q}]} \sum_{v \in T} \sum_{j=1}^{q} \log \frac{\|\mathbf{x}\|_{v} \cdot\left\|L_{j}\right\|_{v}}{\left\|L_{j}(\mathbf{x})\right\|_{v}} \geq(2 m-l+1+2 \varepsilon) h(\mathbf{x})+c_{6} .
$$

Since the set of points $\mathbf{x} \in \mathbb{G}^{m+1}$ with $\varepsilon h(\mathbf{x})+c_{6}<0$ is finite, excluding 
these points yields

$$
\frac{1}{[\mathbb{G}: \mathbb{Q}]} \sum_{v \in T} \sum_{j=1}^{q} \log \frac{\|\mathbf{x}\|_{v} \cdot\left\|L_{j}\right\|_{v}}{\left\|L_{j}(\mathbf{x})\right\|_{v}} \geq(2 m-l+1+\varepsilon) h(\mathbf{x}) .
$$

Thus, the above quoted Theorem B of [RW] implies Theorem 7.

Similarly, by using Theorem 3.1 of [RV] Theorem 2 can be generalized as follows:

THEOREM 8. Let $q, m, l$ be positive integers with $q>2 m-l+1,1 \leq l \leq$ $m+1$. Let $c, \nu$ be real numbers with $c>0, \nu<q-2 m+l-1$ and $\mathbb{G}$ a finite extension of $\mathbb{K}$. For $n=1,2, \ldots$, let $F_{n}(\mathbf{X})=F_{n}\left(X_{0}, \ldots, X_{m}\right) \in O_{S}[\mathbf{X}]$ denote a decomposable form of degree $q$ which factorizes into linear factors over $\mathbb{G}$, and suppose that these factors are in general position for each $n$. Then the points $\mathbf{x}_{n} \in O_{S}^{m+1}, n=1,2, \ldots$, satisfying

$$
0<N_{S}\left(F_{n}\left(\mathbf{x}_{n}\right)\right) \leq c H_{S}\left(\mathbf{x}_{n}\right)^{\nu} \quad \text { for } n=1,2, \ldots,
$$

and

$$
h\left(F_{n}\right)=o\left(h\left(\mathbf{x}_{n}\right)\right) \quad \text { if } h\left(\mathbf{x}_{n}\right) \rightarrow \infty \text { as } n \rightarrow \infty,
$$

are $l$-degenerate (for the concept of $l$-degenerate, see $[\mathrm{RV}]$ ).

Theorems 7 and 8 have applications to resultant inequalities of the same type as Theorems 1 and 2 above.

As was shown earlier, Theorem 1 implies Theorem 4. Similarly, our Theorem 7 has the following consequence.

TheOREM 9. Given positive integers $q, m, l$ with $q>2 m-l+1$, $1 \leq l \leq m+1$, and a polynomial $G(\mathbf{X}) \in O_{S}[\mathbf{X}]$ in $\mathbf{X}=\left(X_{0}, \ldots, X_{m}\right)$ with total degree less than $q-2 m+l-1$. Let $F(\mathbf{X}) \in O_{S}[\mathbf{X}]$ be a decomposable form of degree $q$ whose linear factors are in general position. Then the set of solutions of

$$
F(\mathbf{x})=G(\mathbf{x}) \quad \text { in } \mathbf{x}=\left(x_{0}, \ldots, x_{m}\right) \in O_{S}^{m+1}
$$

is contained in a finite union of linear subspaces of $\mathbb{K}^{m+1}$ of dimension at most l.

\section{References}

[EGy1] J. H. Evertse and K. Győry, Finiteness criteria for decomposable form equations, Acta Arith. 50 (1988), 357-379.

[EGy2] - - - Decomposable form equations, in: New Advances in Transcendence Theory, A. Baker (ed.), Cambridge Univ. Press, 1988, 175-202.

[EGy3] - - - Effective finiteness results for binary forms with given discriminant, Compositio Math. 79 (1991), 169-204. 
[EGy4] J. H. Evertse and K. Győry, The number of families of solutions of decomposable form equations, Acta Arith. 80 (1997), 367-394.

[Gy1] K. Györy, Some applications of decomposable form equations to resultant equations, Colloq. Math. 65 (1993), 267-275.

[Gy2] - On the number of pairs of polynomials with given resultant or given semiresultant, Acta Sci. Math. (Szeged) 57 (1993), 515-529.

[Gy3] -, On the irreducibility of neighbouring polynomials, Acta Arith. 67 (1994), 283-296.

[L] S. Lang, Fundamentals of Diophantine Geometry, Springer, 1983.

[RV] M. Ru and P. Vojta, Schmidt's subspace theorem with moving targets, Invent. Math. 127 (1997), 51-65.

[RW] M. Ru and P. M. Wong, Integral points of $\mathbf{P}^{n}-\{2 n+1$ hyperplanes in general position\}, ibid. 106 (1991), 195-216.

[Schl] H. P. Schlickewei, Inequalities for decomposable forms, Astérisque 41-42 (1977), 267-271

[Sch1] W. M. Schmidt, Inequalities for resultants and for decomposable forms, in: Diophantine Approximation and its Applications, Academic Press, New York, 1973, 235-253.

[Sch2] -, Diophantine Approximation, Lecture Notes in Math. 785, Springer, Berlin, 1980.

[W] E. Wirsing, On approximations of algebraic numbers by algebraic numbers of bounded degree, in: Proc. Sympos. Pure Math. 20, Amer. Math. Soc., Providence, R.I., 1971, 213-247.

Institute of Mathematics and Informatics

Lajos Kossuth University

Pf. 12, H-4010 Debrecen, Hungary

E-mail: gyory@math.klte.hu
Department of Mathematics University of Houston

Houston, Texas 77204

U.S.A.

E-mail: minru@math.uh.edu

Received on 15.9.1997

and in revised form on 2.6.1998 